\title{
Singular Hammerstein-Volterra Integral Equation and Its Numerical Processing
}

\author{
A. M. Al-Bugami \\ Department of Mathematics, Faculty of Sciences, Taif University, Taif, KSA \\ Email: abeer101aa@yahoo.com
}

How to cite this paper: Al-Bugami, A.M. (2021) Singular Hammerstein-Volterra Integral Equation and Its Numerical Processing. Journal of Applied Mathematics and Physics, 9, 379-390.

https://doi.org/10.4236/jamp.2021.92026

Received: January 21, 2021

Accepted: February 23, 2021

Published: February 26, 2021

Copyright $\odot 2021$ by author(s) and Scientific Research Publishing Inc. This work is licensed under the Creative Commons Attribution International License (CC BY 4.0).

\begin{abstract}
In this paper, the existence and uniqueness of solution of singular Hammerstein-Volterra integral equation (H-VIE) are considered. Toeplitz matrix (TMM) and product Nystrom method (PNM) to solve the H-VIE with singular logarithmic kernel are used. The absolute error is calculated.
\end{abstract}

\section{Keywords}

Integral Equation, Hammerstein, Logarithmic Kernel

\section{Introduction}

http://creativecommons.org/licenses/by/4.0/ The singular integral equations are considered to be of more interest than the Open Access others and a close form of solution is generally not available. Therefore, great attention must be considered for the numerical solution of these equations. Abdou in [1], studied Fredholm-Volterra integral equation with singular kernel. Al-Bugami, in [2], studied some numerical methods for solving singular and nonsingular integral equations. Abdou, El-Sayed and Deebs, in [3], obtained a solution of nonlinear integral equation. Also in [4], Abdou and Hendi used numerical solution for solving Fredholm integral equation with Hilbert kernel. In [5], Al-Bugami used TMM and Volterra-Hammerstein integral equation with a generalized singular kernel. In [6], Abdou, Borai, and El-Kojok used TMM and nonlinear integral equation of Hammerstein type. Al-Bugami, in [7], studied the error analysis for numerical solution of HIE with a generalized singular kernel. A. Shahsavaran in [8], studied Lagrange functions method for solving nonlinear F-VIE. In [9], Darwish, studied the nonlinear Fredholm-Volterra integral equations with hysteresis. In [10], Mirzaee used numerical solution of nonlinear F-VIEs via Bell polynomials. In [11], Raad studied linear F-VIE with logarithmic kernel and solved the linear system of Fredholm integral equations numeri- 
cal with logarithmic form.

\section{Existence and Uniqueness of the Solution of H-VIE}

Consider:

$$
\mu \phi(x, t)=f(x, t)+\lambda \int_{-a}^{a} K(x, y) \gamma(y, t, \phi(y, t)) \mathrm{d} y+\lambda \int_{0}^{t} F(t, \tau) \phi(x, \tau) \mathrm{d} \tau
$$

This formula is measured in $L_{2}[-a, a] \times C[0, T], T<\infty$, where the FI term is measured with respect to position. While the VI term is considered in time, and $f(x, t)$ is known function. $\lambda$ is the parameter, while $\mu$ defines the kind of the integral Equation (1).

We assume:

1) $K(x, y) \in C([-a, a] \times[-a, a])$, and satisfies:

$$
\left[\int_{-a}^{a} \int_{-a}^{a}|K(x, y)|^{2} \mathrm{~d} y \mathrm{~d} x\right]^{\frac{1}{2}}=A_{1}<\infty,\left(A_{1} \text { is a constant }\right)
$$

2) $F(t, \tau) \in C([0, T] \times[0, T]), 0 \leq \tau \leq t \leq T \leq \infty$, satisfies:

$$
|F(t, \tau)| \leq A_{2}
$$

3) $f(x, t)$ is continuous in $L_{2}[-a, a] \times C[0, T]$ where:

$$
\|f(x, t)\|=\max _{0 \leq t \leq T} \int_{0}^{t}\left[\int_{a}^{b}|f(x, \tau)|^{2} \mathrm{~d} x\right]^{\frac{1}{2}} \mathrm{~d} \tau=A_{3}
$$

4) $\gamma(x, t, \phi(x, t))$, satisfies for the constant $B>B_{1}, B>p$, the following conditions:

a) $\int_{0}^{t} \int_{a}^{b}\left(|\gamma(x, t, \phi(x, t))|^{2} \mathrm{~d} x \mathrm{~d} t\right)^{\frac{1}{2}} \leq B_{1}\|\phi(x, t)\|_{L_{2}[a, b] \times C[0, T]}$

b) $\left\|\gamma\left(x, t, \phi_{1}(x, t)\right)-\gamma\left(x, t, \phi_{2}(x, t)\right)\right\| \leq N(x, t)\left|\phi_{1}(x, t)-\phi_{2}(x, t)\right|$

where $\|N(x, t)\|_{L_{2}[a, b] \times C[0, T]}=p$

In other words, we prove that the solution exists using the successive approximation method, also called the Picard method, that we pick up any real continuous function $\phi_{0}(x, t)$ in $L_{2}[-a, a] \times C[0, T]$, we assume $\phi_{0}(x, t)=f(x, t)$, then construct a sequence $\phi_{n}$ defined by

$$
\begin{aligned}
\phi_{n}(x, t)= & f(x, t)+\lambda \int_{-a}^{a} K(x, y) \gamma\left(y, t, \phi_{n-1}(y, t)\right) \mathrm{d} y \\
& +\lambda \int_{0}^{t} F(t, \tau) \phi_{n-1}(x, \tau) \mathrm{d} \tau,(\mu=1) \\
\phi_{n-1}(x, t)= & f(x, t)+\lambda \int_{-a}^{a} K(x, y) \gamma\left(y, t, \phi_{n-2}(y, t)\right) \mathrm{d} y \\
& +\lambda \int_{0}^{t} F(t, \tau) \phi_{n-2}(x, \tau) \mathrm{d} \tau,(\mu=1)
\end{aligned}
$$




$$
\begin{aligned}
\psi_{n}(x, t)= & \phi_{n}(x, t)-\phi_{n-1}(x, t) \\
= & \lambda \int_{-a}^{a} K(x, y)\left[\gamma\left(y, t, \phi_{n-1}(y, t)\right)-\gamma\left(y, t, \phi_{n-2}(y, t)\right)\right] \mathrm{d} y \\
& +\lambda \int_{0}^{t} F(t, \tau)\left[\phi_{n-1}(x, \tau)-\phi_{n-2}(x, \tau)\right] \mathrm{d} \tau, n=1,2, \cdots
\end{aligned}
$$

Then:

$$
\phi_{n}(x, t)=\sum_{i=0}^{n} \psi_{i}(x, t)
$$

Hence

$$
\psi_{n}(x, t)=f(x, t)+\lambda \int_{-a}^{a} K(x, y) \gamma\left(y, t, \psi_{n-1}(y, t)\right) \mathrm{d} y+\lambda \int_{0}^{t} F(t, \tau) \psi_{n-1}(x, \tau) \mathrm{d} \tau
$$

Using the properties of the norm, we obtain:

$$
\left\|\psi_{n}(x, t)\right\| \leq|\lambda|\left\|\int_{-a}^{a} K(x, y) \gamma\left(y, t, \psi_{n-1}(y, t)\right) \mathrm{d} y\right\|+|\lambda|\left\|\int_{0}^{t} F(t, \tau) \psi_{n-1}(x, \tau) \mathrm{d} \tau\right\|
$$

For $n=1$, we get

$$
\begin{aligned}
\left\|\psi_{1}(x, t)\right\| \leq & \leq|\lambda|\left\|\int_{-a}^{a} K(x, y) \gamma\left(y, t, \psi_{0}(y, t)\right) \mathrm{d} y\right\|+|\lambda|\left\|\int_{0}^{t} F(t, \tau) \psi_{0}(x, \tau) \mathrm{d} \tau\right\| \\
& \left.\leq|\lambda| \| \int_{-a}^{a}|K(x, y)|^{2} \mathrm{~d} y\right)^{\frac{1}{2}}\left(\int_{-a}^{a}\left|\gamma\left(y, t, \psi_{0}(y, t)\right)\right|^{2} \mathrm{~d} y\right)^{\frac{1}{2}} \| \\
& +|\lambda|\left\|\int _ { 0 } ^ { t } \left|F(t, \tau)\left\|\psi_{0}(x, \tau) \mid \mathrm{d} \tau\right\|\right.\right.
\end{aligned}
$$

Using Cauchy Schwarz inequality and from conditions (i)-(iv-a) with $\psi_{0}=f(x, t)$ and $\|f\|=A_{3}$, we get

$$
\begin{aligned}
\left\|\psi_{1}(x, t)\right\| \leq & |\lambda| \max \int_{0}^{t}\left[\int_{-a}^{a}\left(\int_{-a}^{a}|K(x, y)|^{2} \mathrm{~d} y \int_{-a}^{a}\left|\gamma\left(y, t, \psi_{0}(y, t)\right)\right|^{2} \mathrm{~d} y\right) \mathrm{d} x\right]^{\frac{1}{2}} \mathrm{~d} \tau \\
& +|\lambda| A_{2} \int_{0}^{t}\left\|\psi_{0}(x, \tau)\right\| \mathrm{d} \tau \\
\leq & |\lambda| A_{1} A_{3} B_{1}+|\lambda| A_{2} A_{3}\|t\|
\end{aligned}
$$

We have $0 \leq \tau \leq t \leq T \leq \infty$, then $\max |t|=T=L$, and then we have:

$$
\left\|\psi_{1}(x, t)\right\| \leq|\lambda| A_{3}\left(A_{1} B_{1}+A_{2} L\right)
$$

In general, we get:

$$
\left\|\psi_{1}(x, t)\right\| \leq|\lambda|^{n} A_{3}\left(A_{1} B_{1}+A_{2} L\right)^{n}=A_{3} \alpha^{n}, \alpha=|\lambda|\left(A_{1} B_{1}+A_{2} L\right)
$$

This bound makes the sequence $\psi_{n}(x, t)$ converges if

$$
\alpha<1 \Rightarrow|\lambda|<\frac{1}{A_{1} B_{1}+A_{2} L}
$$


The result (4), leads us to say that the formula (2) has a convergent solution. So let $n \rightarrow \infty$, we have:

$$
\phi(x, t)=\sum_{i=0}^{\infty} \psi_{i}(x, t)=\frac{A_{3}}{1-\alpha}, \quad(\alpha<1)
$$

The infinite series of (5) is convergent, and $\phi(x, t)$ represents the convergent solution of Equation (1). Also each of $\psi_{i}$ is continuous, therefore $\phi(x, t)$ is also continuous.

To show that $\phi(x, t)$ is unique, we assume that $\bar{\phi}(x, t)$ is also a continuous solution of (1) then, we write

$$
\begin{aligned}
\phi(x, t)-\bar{\phi}(x, t)= & \lambda \int_{-a}^{a} K(x, y)[\gamma(y, t, \phi(y, t))-\gamma(y, t, \bar{\phi}(y, t))] \mathrm{d} y \\
& +\lambda \int_{0}^{t} F(t, \tau)[\phi(x, \tau)-\bar{\phi}(x, \tau)] \mathrm{d} \tau,(\mu=1)
\end{aligned}
$$

which leads us to the following:

$$
\begin{aligned}
\|\phi(x, t)-\bar{\phi}(x, t)\| \leq & |\lambda|\left\|\int_{-a}^{a}|K(x, y)||\gamma(y, t, \phi(y, t))-\gamma(y, t, \bar{\phi}(y, t))| \mathrm{d} y\right\| \\
& +|\lambda|\left\|\int_{0}^{t}|F(t, \tau)||\phi(x, \tau)-\bar{\phi}(x, \tau)| \mathrm{d} \tau\right\|
\end{aligned}
$$

Using conditions (iv-b), then we have:

$$
\begin{aligned}
& \|\phi(x, t)-\bar{\phi}(x, t)\| \\
& \leq|\lambda| \max _{0 \leq t \leq T} \int_{0}^{t}\left[\int_{-a}^{a} \int_{-a}^{a}(|K(x, y)| \mathrm{d} x \mathrm{~d} y)^{\frac{1}{2}}\left(\int_{-a}^{a} N^{2}(x, t)|\phi(x, t)-\bar{\phi}(x, t)|^{2} \mathrm{~d} y\right)^{\frac{1}{2}}\right] \mathrm{d} \tau \\
& +|\lambda|\left\|\int_{0}^{t}|F(t, \tau)||\phi(x, t)-\bar{\phi}(x, t)| \mathrm{d} \tau\right\|
\end{aligned}
$$

Finally, with the aid of conditions (i) and (ii):

$$
\|\phi(x, t)-\bar{\phi}(x, t)\| \leq \alpha\|\phi(x, t)-\bar{\phi}(x, t)\|
$$

Then:

$$
(1-\alpha)\|\phi(x, t)-\bar{\phi}(x, t)\| \leq 0
$$

Since $\|\phi(x, t)-\bar{\phi}(x, t)\|$ is necessarily non-negative, and $\alpha<1$ :

$$
\|\phi(x, t)-\bar{\phi}(x, t)\|=0 \Rightarrow \phi(x, t)=\bar{\phi}(x, t)
$$

It follows that if (1) has a solution it must be unique.

\section{SHIEs}

Consider:

$$
\phi(x, t)=f(x, t)+\lambda \int_{-a}^{a} K(x, y) \gamma(y, t, \phi(y, t)) \mathrm{d} y+\lambda \int_{0}^{t} F(t, \tau) \phi(x, \tau) \mathrm{d} \tau
$$

when $t=0$ Equation (13) becomes: 


$$
\phi_{0}(x)=f_{0}(x)+\lambda \int_{-a}^{a} K(x, y) \gamma\left(y, \phi_{0}(y)\right) \mathrm{d} y
$$

where $\phi_{0}(x)=\phi(x, 0), f_{0}(x)=f(x, 0)$.

The formula (7) represents HIE of the second kind at $t=0$. Divide the interval $[0, T], 0 \leq t \leq T<\infty$ as $0=t_{0} \leq t_{1}<\cdots<t_{k}<\cdots<t_{N}=T$, then using the quadrature formula, the Volterra integral term in (6) becomes:

$$
\int_{0}^{t_{k}} F(t, \tau) \phi(x, \tau) \mathrm{d} \tau=\sum_{j=0}^{k} u_{j} F\left(t_{k}, t_{j}\right) \phi\left(x, t_{j}\right)+o\left(\hbar_{i}^{\tilde{p}+1}\right),\left(\hbar_{k} \rightarrow 0, \tilde{p}>0\right)
$$

where $\hbar_{k}=\max _{0 \leq j \leq k} h_{j}, \quad h_{j}=t_{j+1}-t_{j}$

Using (8) in (6), we have:

$$
\phi_{k}(x)=f_{k}(x)+\lambda \int_{-a}^{a} K(x, y) \gamma\left(y, t_{k}, \phi_{k}(y)\right) \mathrm{d} y+\lambda \sum_{j=0}^{k} u_{j} F_{k j} \phi_{j}(x)
$$

where $\phi_{k}(x)=\phi\left(x, t_{k}\right), f_{k}(x)=f\left(x, t_{k}\right), F_{k j}=F\left(t_{k}, t_{j}\right)$.

$$
\mu_{n} \phi_{n}(x)=G_{n}(x)+\lambda \int_{-a}^{a} K(x, y) \phi_{n}(y) \mathrm{d} y
$$

where $\mu_{n}=1-\lambda F_{n n} u_{n}, G_{n}(x)=f_{n}(x)+\lambda \sum^{n-1} u_{j} F_{n j} \gamma\left(x, t_{j}, \phi_{j}(x)\right), n=0,1, \cdots, N$.

The formula (10) represents SHIEs of the second kind, and we have $N$ unknown $\phi_{n}(x)$.

\section{Some Numerical Techniques for Solving SHIEs}

\subsection{The TMM}

In this section, we present the TMM to obtain numerical solution for HIE of the second kind with singular kernel. Consider:

$$
\phi(x)=f(x)+\lambda \int_{-a}^{a} K(|x-y|) \gamma(y, \phi(y)) \mathrm{d} y
$$

Write the integral term in the form:

$$
\int_{-a}^{a} K(|x-y|) \gamma(y, \phi(y)) \mathrm{d} y=\sum_{n=-N}^{N-1} \int_{n h}^{n h+h} K(|x-y|) \gamma(y, \phi(y)) \mathrm{d} y,\left(h=\frac{2 a}{N}\right)
$$

Approximate the integral in the right hand side of Equation (12) by:

$$
\begin{aligned}
& \int_{n h}^{n h+h} K(|x-y|) \gamma(y, \phi(y)) \mathrm{d} y \\
& =A_{n}(x) \gamma(n h, \phi(n h))+B_{n}(x) \gamma(n h+h, \phi(n h+h))+R
\end{aligned}
$$

where $A_{n}(x)$ and $B_{n}(x)$ are two arbitrary functions. Putting $\phi(x)=1, x$ in Equation (13), where in this case we choose $R=0$. By solving the result, then we take:

$$
A_{n}(x)=\frac{1}{h}[\gamma(n h+h, n h+h) I(x)-\gamma(n h+h, 1) J(x)]
$$

And

$$
B_{n}(x)=\frac{1}{h}[\gamma(n h+h, 1) J(x)-\gamma(n h, n h) I(x)]
$$


where:

$$
\begin{gathered}
I(x)=\int_{n h}^{n h+h} K(|x-y|) \gamma(y, 1) \mathrm{d} y \\
J(x)=\int_{n h}^{n h+h} K(|x-y|) \gamma(y, y) \mathrm{d} y
\end{gathered}
$$

The relation (12), becomes:

$$
\int_{-a}^{a} K(|x-y|) \gamma(y, \phi(y)) \mathrm{d} y=\sum_{n=-N}^{N} D_{n}(x) \gamma(n h, \phi(n h))
$$

where

$$
D_{n}(x)=\left\{\begin{array}{lrl}
A_{-N}(x), & n & =-N \\
A_{n}(x)+B_{n}(x), & -N & <n<N \\
B_{N-1}(x), & n & =N
\end{array}\right.
$$

The IE (11) becomes:

$$
\phi(x)-\lambda \sum_{n=-N}^{N} D_{n}(x) \gamma(n h, \phi(n h))=f(x)
$$

Putting $x=m h$, we have:

$$
\phi_{m}-\lambda \sum_{n=-N}^{N} D_{n, m} \gamma_{n}\left(\phi_{n}\right)=f_{m},-N \leq m \leq N
$$

where $\phi_{m}=\phi(m h), D_{n, m}=D_{n}(m h), f_{m}=f(m h)$.

The matrix $D_{n, m}$ may be written as $D_{n, m}=G_{n, m}+E_{n, m}$, where:

$$
G_{n, m}=A_{n}(m h)+B_{n-1}(m h),-N \leq n, m \leq N
$$

Is a Toeplitz matrix of order $2 N+1$ and:

$$
E_{n, m}(x)=\left\{\begin{array}{lc}
B_{-N-1}(x), & n=-N, m=-N+i \\
0, & -N<n<N \\
A_{N}(x), & n=N, m=-N+i
\end{array}\right.
$$

where $0 \leq i \leq 2 n$. The solution of the formula (20):

$$
\phi_{m}=\left[I-\lambda\left(G_{n, m}+E_{n, m}\right)\right]^{-1} f_{m},\left|I-\lambda\left(G_{n, m}+E_{n, m}\right)\right| \neq 0
$$

Also

$$
R=\left|\int_{-a}^{a} K(|x-y|) \gamma(y, \phi(y)) \mathrm{d} y-\sum_{n=-N}^{N} D_{n m} \gamma(n h, \phi(n h))\right|
$$

\subsection{The PNM}

Consider:

$$
\phi(x)-\lambda \int_{-a}^{a} p(x, y) \bar{K}(x, y) \gamma(y, \phi(y)) \mathrm{d} y=f(x)
$$

where $p$ and $\bar{K}$ are badly behaved and well-behaved functions of their arguments, respectively. Then, we get: 


$$
\phi\left(x_{i}\right)-\lambda \sum_{j=0}^{N} w_{i j} \bar{K}\left(x_{i}, y_{j}\right) \gamma\left(y_{j}, \phi\left(y_{j}\right)\right)=f\left(x_{i}\right)
$$

where $x_{i}=y_{i}=a+i h, i=0,1, \cdots, N$ with $h=\frac{2 a}{N}, N$ even and $w_{i j}$ are the weights. When $x=x_{i}$, we write:

$$
\int_{-a}^{a} p\left(x_{i}, y\right) \bar{K}\left(x_{i}, y\right) \gamma(y, \phi(y)) \mathrm{d} y=\sum_{j=0}^{\frac{N-2}{2}} \int_{y_{2 j}}^{y_{2 j+2}} p\left(x_{i}, y\right) \bar{K}\left(x_{i}, y\right) \gamma(y, \phi(y)) \mathrm{d} y
$$

Form relation (25) through (27) we find:

$$
\sum_{j=0}^{N} w_{i j} \bar{K}\left(x_{i}, y_{j}\right) \gamma\left(y_{j}, \phi\left(y_{j}\right)\right)=\sum_{j=0}^{\frac{N-2}{2}} \int_{y_{2 j}}^{y_{2 j+2}} p\left(x_{i}, y\right) \bar{K}\left(x_{i}, y\right) \gamma(y, \phi(y)) \mathrm{d} y
$$

Then, we obtain:

$$
\begin{aligned}
& \int_{-a}^{a} p\left(x_{i}, y\right) \bar{K}\left(x_{i}, y\right) \gamma(y, \phi(y)) \mathrm{d} y \\
& =\sum_{j=0}^{\frac{N-2}{2} y_{2 j+2}} p\left(x_{i}, y\right)\left\{\frac{\left(y_{2 j+1}-y\right)\left(y_{2 j+2}-y\right)}{2 h^{2}} \gamma\left(y_{2 j}, \phi\left(y_{2 j}\right)\right)\right. \\
& +\frac{\left(y-y_{2 j}\right)\left(y_{2 j+2}-y\right)}{h^{2}} \gamma\left(y_{2 j+1}, \phi\left(y_{2 j+1}\right)\right) \\
& \left.+\frac{\left(y_{2 j+1}-y\right)\left(y_{2 j}-y\right)}{2 h^{2}} \gamma\left(y_{2 j+2}, \phi\left(y_{2 j+2}\right)\right)\right\} \mathrm{d} y
\end{aligned}
$$

Therefore:

$$
\begin{array}{ll}
w_{i, 0}=\beta_{1}\left(y_{i}\right) & w_{i, 2 j+1}=2 \gamma_{j+1}\left(y_{i}\right) \\
w_{i, 2 j}=\alpha_{i}\left(y_{i}\right)+\beta_{j+1}\left(y_{i}\right) & w_{i, N}\left(y_{i}\right)=\alpha_{\frac{N}{2}}\left(y_{i}\right)
\end{array}
$$

where:

$$
\begin{aligned}
& \alpha_{j}\left(y_{i}\right)=\frac{1}{2 h^{2}} \int_{y_{2 j-2}}^{y_{2 j}} p\left(y_{i}, y\right)\left(y-y_{2 j-2}\right)\left(y-y_{2 j-1}\right) \mathrm{d} y \\
& \beta_{j}\left(y_{i}\right)=\frac{1}{2 h^{2}} \int_{y_{2 j-2}}^{y_{2 j}} p\left(y_{i}, y\right)\left(y-y_{2 j-1}\right)\left(y-y_{2 j}\right) \mathrm{d} y \\
& \gamma_{j}\left(y_{i}\right)=\frac{1}{2 h^{2}} \int_{y_{2 j-2}}^{y_{2 j}} p\left(y_{i}, y\right)\left(y-y_{2 j-2}\right)\left(y_{2 j}-y\right) \mathrm{d} y
\end{aligned}
$$

We now introduce the change of variable $y=y_{2 j-2}+\zeta h, 0 \leq \zeta \leq 2$ thus the system (30) becomes:

$$
\begin{gathered}
\alpha_{j}\left(y_{i}\right)=\frac{h}{2} \int_{0}^{2} \zeta(\zeta-1) p\left(y_{2 j-2}+\zeta h, y_{i}\right) \mathrm{d} \zeta \\
\beta_{j}\left(y_{i}\right)=\frac{h}{2} \int_{0}^{2}(\zeta-1)(\zeta-2) p\left(y_{2 j-2}+\zeta h, y_{i}\right) \mathrm{d} \zeta
\end{gathered}
$$




$$
\gamma_{j}\left(y_{i}\right)=\frac{h^{2}}{2} \int_{0}^{\zeta} \zeta(2-\zeta) p\left(y_{2 j-2}+\zeta h, y_{i}\right) \mathrm{d} \zeta
$$

If we define:

$$
\psi_{i}=\int_{0}^{2} \zeta^{i} p\left(y_{2 j-2}+\zeta h, y_{i}\right) \mathrm{d} \zeta
$$

For $p(x, y)=p(x-y)$, we have:

$$
\psi_{i}=\int_{0}^{2} \zeta^{i} p\left(y_{i}, y_{2 j-2}+\zeta h\right) \mathrm{d} \zeta, i=0,1,2
$$

When $y_{i}-y_{2 j-2}=(i-2 j+2) h$. If we assume $z=i-2 j+2$, then:

$$
\begin{aligned}
& \alpha_{j}\left(y_{i}\right)=\frac{h}{2} \int_{0}^{2} \zeta(\zeta-1) p(z-\zeta) \mathrm{d} \zeta \\
& \beta_{j}\left(y_{i}\right)=\frac{h}{2} \int_{0}^{2}(\zeta-1)(\zeta-2) p(z-\zeta) \mathrm{d} \zeta \\
& \gamma_{j}\left(y_{i}\right)=\frac{h}{2} \int_{0}^{2} \zeta(2-\zeta) p(z-\zeta) \mathrm{d} \zeta
\end{aligned}
$$

Hence, the system (29) becomes:

$$
\begin{array}{cc}
w_{i, 0}=\frac{h}{2}\left[2 \psi_{0}(z)-3 \psi_{1}(z)+\psi_{2}(z)\right], & z=i \\
w_{i, 2 j+1}=h\left[2 \psi_{1}(z)-\psi_{2}(z)\right], \quad z=i-2 j & \\
w_{i, 2 j}=\frac{h}{2}\left[\psi_{2}(z)-\psi_{1}(z)+2 \psi_{0}(z-2)-3 \psi_{1}(z-2)+\psi_{2}(z-2)\right], & z=i-2 j+2 \\
w_{i, N}=\frac{h}{2}\left[\psi_{2}(z)-\psi_{1}(z)\right], & z=i-N+2
\end{array}
$$

Therefore, the integral Equation (25) is reduced to SLAEs as in (26) or: $(I-\lambda W) \phi=F$

Which has the solution:

$$
\phi=(I-\lambda W)^{-1} F,|I-\lambda W| \neq 0
$$

The PNM is said to be convergent of order $r$ in $[-a, a]$. If for $N$ sufficiently large, there exists a constant $C>0$ independent of $N$ such that:

$$
\left\|\phi(x)-\phi_{N}(x)\right\| \leq C N^{-r}
$$

\section{Numerical Applications}

We using TMM and PNM at $N=20,40, T=0.03,0.7, \lambda=1$, and $\mu=1$. In Tables 1-4:

$\phi_{\text {Exact }} \rightarrow$ Exact solution, $\phi_{T} \rightarrow$ appro. sol. of TMM, $E_{T} \rightarrow$ the absolute error of TMM, $\phi_{N} \rightarrow$ appro. sol. of PNM, $E_{N} \rightarrow$ the absolute error of PNM.

Example 1

Consider:

$$
\phi(x, t)=f(x, t)+\lambda \int_{-1}^{1} \ln |x-y|(y t)^{2} \mathrm{~d} y+\lambda \int_{0}^{t} \tau^{2} \phi(x, \tau) \mathrm{d} \tau
$$


Table 1. The values of exact, approximate solutions, and errors by using TMM, PNM at $N=20$.

\begin{tabular}{|c|c|c|c|c|c|c|}
\hline$T$ & $x$ & $\phi_{\text {Exact }}$ & $\phi_{T}$ & $E_{T}$ & $\phi_{N}$ & $E_{N}$ \\
\hline \multirow{11}{*}{0.03} & -1.0 & -0.03000000 & -0.030701591 & $7.0159 \mathrm{E}-4$ & -0.03002738 & $2.7386 \mathrm{E}-5$ \\
\hline & -0.8 & -0.02400000 & -0.023838516 & $1.6148 \mathrm{E}-4$ & -0.02403073 & $3.0733 \mathrm{E}-5$ \\
\hline & -0.6 & -0.01800000 & -0.017855743 & $1.4425 \mathrm{E}-4$ & -0.01803683 & $3.6835 \mathrm{E}-5$ \\
\hline & -0.4 & -0.01200000 & -0.011903314 & $9.6685 \mathrm{E}-5$ & -0.01203176 & $3.1761 \mathrm{E}-5$ \\
\hline & -0.2 & -0.00600000 & -0.005955438 & $4.4561 \mathrm{E}-5$ & -0.00632818 & $3.2818 \mathrm{E}-5$ \\
\hline & 0 & 0 & 0.000000452 & $4.5279 \mathrm{E}-7$ & -0.00002599 & $2.5995 \mathrm{E}-5$ \\
\hline & 0.2 & 0.006000000 & 0.0059710686 & $2.8931 \mathrm{E}-5$ & 0.005976067 & $2.3932 \mathrm{E}-5$ \\
\hline & 0.4 & 0.012000000 & 0.0011959300 & $4.6995 \mathrm{E}-5$ & 0.011983505 & $1.6494 \mathrm{E}-5$ \\
\hline & 0.6 & 0.018000000 & 0.0179646910 & $3.5308 \mathrm{E}-5$ & 0.017987883 & $1.2116 \mathrm{E}-5$ \\
\hline & 0.8 & 0.024000000 & 0.0239827313 & $1.7268 \mathrm{E}-5$ & 0.023995269 & $4.7302 \mathrm{E}-6$ \\
\hline & 1.0 & 0.030000000 & 0.029999603 & $3.9617 \mathrm{E}-7$ & 0.030003419 & $3.4192 \mathrm{E}-6$ \\
\hline \multirow{11}{*}{0.7} & -1.0 & -0.70000000 & -0.716358582 & $1.6358 \mathrm{E}-2$ & -0.70031893 & $3.1893 \mathrm{E}-4$ \\
\hline & -0.8 & -0.56000000 & -0.556088118 & $3.9118 \mathrm{E}-3$ & -0.56033293 & 3.3293E-4 \\
\hline & -0.6 & -0.42000000 & -0.416502750 & $3.4972 \mathrm{E}-3$ & -0.42054763 & $5.4763 \mathrm{E}-4$ \\
\hline & -0.4 & -0.28000000 & -0.277643540 & $2.3564 \mathrm{E}-3$ & -0.28051997 & $5.1997 \mathrm{E}-4$ \\
\hline & -0.2 & -0.14000000 & -0.138874201 & $1.1257 \mathrm{E}-3$ & -0.14061900 & $6.1900 \mathrm{E}-4$ \\
\hline & 0 & 0 & 0.0001142305 & $1.1423 \mathrm{E}-4$ & -0.00050225 & $5.0225 \mathrm{E}-4$ \\
\hline & 0.2 & 0.140000000 & 0.1394831440 & $5.1685 \mathrm{E}-4$ & 0.139540294 & $4.5979 \mathrm{E}-4$ \\
\hline & 0.4 & 0.280000000 & 0.2792953877 & $7.0461 \mathrm{E}-4$ & 0.279740200 & $2.5979 \mathrm{E}-4$ \\
\hline & 0.6 & 0.420000000 & 0.4195246008 & $4.7539 \mathrm{E}-4$ & 0.419885056 & $1.1494 \mathrm{E}-4$ \\
\hline & 0.8 & 0.560000000 & 0.5600309299 & $3.0929 \mathrm{E}-5$ & 0.560081782 & $8.1782 \mathrm{E}-4$ \\
\hline & 1.0 & 0.700000000 & 0.7003734045 & $3.7340 \mathrm{E}-4$ & 0.700159944 & $1.5994 \mathrm{E}-4$ \\
\hline
\end{tabular}

Table 2. The values of exact, approximate solutions, and errors by using TMM, PNM at $N=40$.

\begin{tabular}{|c|c|c|c|c|c|c|}
\hline$T$ & $x$ & $\phi_{\text {Exact }}$ & $\phi_{T}$ & $E_{T}$ & $\phi_{N}$ & $E_{N}$ \\
\hline \multirow{11}{*}{0.03} & -1.0 & -0.03000000 & -0.031354257 & $1.3542 \mathrm{E}-3$ & -0.03002817 & $2.8170 \mathrm{E}-5$ \\
\hline & -0.8 & -0.02400000 & -0.023848333 & $1.5156 \mathrm{E}-4$ & -0.02403524 & $3.5249 \mathrm{E}-5$ \\
\hline & -0.6 & -0.01800000 & -0.017860806 & $1.3919 \mathrm{E}-4$ & -0.01803612 & $3.6128 \mathrm{E}-5$ \\
\hline & -0.4 & -0.01200000 & -0.011906018 & $9.3981 \mathrm{E}-5$ & -0.01203478 & $3.4780 \mathrm{E}-5$ \\
\hline & -0.2 & -0.00600000 & -0.005956673 & $4.3326 \mathrm{E}-5$ & -0.00603193 & $3.1939 \mathrm{E}-5$ \\
\hline & 0 & 0 & 0.0000002134 & $2.1349 \mathrm{E}-7$ & -0.00002798 & $2.7985 \mathrm{E}-5$ \\
\hline & 0.2 & 0.006000000 & 0.005971517 & $2.8482 \mathrm{E}-5$ & 0.059768287 & $2.3171 \mathrm{E}-5$ \\
\hline & 0.4 & 0.012000000 & 0.0119602026 & $3.9797 \mathrm{E}-5$ & 0.011982306 & $1.7693 \mathrm{E}-5$ \\
\hline & 0.6 & 0.018000000 & 0.0179658449 & $3.4155 \mathrm{E}-5$ & 0.017988279 & $1.1720 \mathrm{E}-5$ \\
\hline & 0.8 & 0.024000000 & 0.0239839397 & $1.6060 \mathrm{E}-5$ & 0.0239946215 & $5.3784 \mathrm{E}-6$ \\
\hline & 1.0 & 0.030000000 & 0.0300003812 & $3.8126 \mathrm{E}-7$ & 0.030002272 & $2.2728 \mathrm{E}-6$ \\
\hline \multirow{11}{*}{0.7} & -1.0 & -0.70000000 & -0.731583858 & $3.1585 \mathrm{E}-2$ & -0.700337200 & $3.3720 \mathrm{E}-4$ \\
\hline & -0.8 & -0.56000000 & -0.556312874 & $3.6871 \mathrm{E}-3$ & -0.560438272 & $4.3827 \mathrm{E}-4$ \\
\hline & -0.6 & -0.42000000 & -0.416615893 & $3.3841 \mathrm{E}-3$ & -0.420531161 & $5.3116 \mathrm{E}-4$ \\
\hline & -0.4 & -0.28000000 & -0.277703301 & $2.2966 \mathrm{E}-3$ & -0.280590379 & $5.9037 \mathrm{E}-4$ \\
\hline & -0.2 & -0.14000000 & -0.138901367 & $1.0986 \mathrm{E}-3$ & -0.140598518 & $5.9851 \mathrm{E}-4$ \\
\hline & 0 & 0 & 0.0001086431 & $1.0864 \mathrm{E}-4$ & -0.000548653 & $5.4865 \mathrm{E}-4$ \\
\hline & 0.2 & 0.140000000 & 0.1394919213 & $5.0807 \mathrm{E}-4$ & 0.1395580310 & $4.4196 \mathrm{E}-4$ \\
\hline & 0.4 & 0.280000000 & 0.2793130480 & $6.8695 \mathrm{E}-4$ & 0.2791722263 & $2.8777 \mathrm{E}-4$ \\
\hline & 0.6 & 0.420000000 & 0.4195464227 & $4.5357 \mathrm{E}-4$ & 0.4198942815 & $1.0571 \mathrm{E}-4$ \\
\hline & 0.8 & 0.560000000 & 0.5600523036 & $5.2303 \mathrm{E}-5$ & 0.560066655 & $6.6665 \mathrm{E}-5$ \\
\hline & 1.0 & 0.700000000 & 0.7003829896 & $3.8298 \mathrm{E}-4$ & 0.7001331775 & $1.3317 \mathrm{E}-4$ \\
\hline
\end{tabular}


Table 3. The values of exact, approximate solutions, and errors by using TMM, PNM at $N=20$.

\begin{tabular}{|c|c|c|c|c|c|c|}
\hline$T$ & $x$ & $\phi_{\text {Exact }}$ & $\phi_{T}$ & $E_{T}$ & $\phi_{N}$ & $E_{N}$ \\
\hline \multirow{11}{*}{0.03} & -1.0 & -0.015000000 & -0.015350899 & $3.5089 \mathrm{E}-4$ & -0.0150137693 & $1.3789 \mathrm{E}-5$ \\
\hline & -0.8 & -0.012000000 & -0.119193964 & $8.0603 \mathrm{E}-5$ & -0.0120155049 & $1.5504 \mathrm{E}-5$ \\
\hline & -0.6 & -0.009000000 & -0.008927989 & $7.2013 \mathrm{E}-5$ & -0.0090185327 & $1.8532 \mathrm{E}-5$ \\
\hline & -0.4 & -0.006000000 & -0.005951798 & $4.8260 \mathrm{E}-5$ & -0.0060159635 & $1.5963 \mathrm{E}-5$ \\
\hline & -0.2 & -0.003000000 & -0.002977777 & $2.2222 \mathrm{E}-5$ & -0.0030164679 & $1.6467 \mathrm{E}-5$ \\
\hline & 0 & 0 & -0.176673371 & $1.7667 \mathrm{E}-7$ & -0.0001304779 & $1.3047 \mathrm{E}-5$ \\
\hline & 0.2 & 0.0030000000 & 0.0029854757 & $1.4524 \mathrm{E}-5$ & 0.00298797512 & $1.2024 \mathrm{E}-5$ \\
\hline & 0.4 & 0.0060000000 & 0.0059795673 & $2.0432 \mathrm{E}-5$ & 0.00599166981 & $8.3301 \mathrm{E}-6$ \\
\hline & 0.6 & 0.0090000000 & 0.0089822306 & $1.7769 \mathrm{E}-5$ & 0.00899382675 & $6.1732 \mathrm{E}-6$ \\
\hline & 0.8 & 0.0120000000 & 0.0119912274 & $8.7725 \mathrm{E}-6$ & 0.01199749658 & $2.5034 \mathrm{E}-6$ \\
\hline & 1.0 & 0.0150000000 & 0.0149997058 & $2.9414 \mathrm{E}-7$ & 0.01500161353 & $1.6135 \mathrm{E}-6$ \\
\hline \multirow{11}{*}{0.7} & -1.0 & -0.350000000 & -0.358222414 & $8.2224 \mathrm{E}-3$ & -0.3502016929 & $2.0169 \mathrm{E}-4$ \\
\hline & -0.8 & -0.280000000 & -0.278111233 & $1.8887 \mathrm{E}-3$ & -0.2802337016 & $2.3370 \mathrm{E}-4$ \\
\hline & -0.6 & -0.210000000 & -0.208307768 & $1.6922 \mathrm{E}-3$ & -0.2103304274 & $3.3042 \mathrm{E}-4$ \\
\hline & -0.4 & -0.140000000 & -0.138862748 & $1.1372 \mathrm{E}-3$ & -0.1403011727 & $3.0117 \mathrm{E}-4$ \\
\hline & -0.2 & -0.700000000 & -0.069466949 & $5.3305 \mathrm{E}-4$ & -0.0703395348 & $3.3953 \mathrm{E}-4$ \\
\hline & 0 & 0 & 0.0000300394 & $3.0039 \mathrm{E}-5$ & -0.000278348 & $2.7834 \mathrm{E}-4$ \\
\hline & 0.2 & 0.7000000000 & 0.069707680 & $2.9231 \mathrm{E}-4$ & 0.0697361145 & $2.6388 \mathrm{E}-4$ \\
\hline & 0.4 & 0.1400000000 & 0.1395986121 & $4.0138 \mathrm{E}-4$ & 0.1398209052 & $1.7909 \mathrm{E}-4$ \\
\hline & 0.6 & 0.2100000000 & 0.209637503 & $3.0262 \mathrm{E}-4$ & 0.209873904 & $1.2609 \mathrm{E}-4$ \\
\hline & 0.8 & 0.2800000000 & 0.279932202 & $6.7797 \mathrm{E}-5$ & 0.2799576210 & $4.2379 \mathrm{E}-4$ \\
\hline & 1.0 & 0.3500000000 & 0.3501244044 & $1.2440 \mathrm{E}-4$ & 0.3500176757 & $1.7675 \mathrm{E}-5$ \\
\hline
\end{tabular}

Table 4. The values of exact, approximate solutions, and errors by using TMM, PNM at $N=40$.

\begin{tabular}{|c|c|c|c|c|c|c|}
\hline$T$ & $x$ & $\phi_{\text {Exact }}$ & $\phi_{T}$ & $E_{T}$ & $\phi_{N}$ & $E_{N}$ \\
\hline \multirow{11}{*}{0.03} & -1.0 & -0.015000000 & -0.015677228 & $6.7722 \mathrm{E}-4$ & -0.015014180 & $1.4180 \mathrm{E}-5$ \\
\hline & -0.8 & -0.012000000 & -0.011924354 & $7.5645 \mathrm{E}-5$ & -0.120177632 & $1.7763 \mathrm{E}-5$ \\
\hline & -0.6 & -0.009000000 & -0.008930517 & $6.9482 \mathrm{E}-5$ & -0.009018179 & $1.8179 \mathrm{E}-5$ \\
\hline & -0.4 & -0.006000000 & -0.005953091 & $4.6908 \mathrm{E}-5$ & -0.006017473 & $1.7473 \mathrm{E}-5$ \\
\hline & -0.2 & -0.003000000 & -0.002978395 & $2.1604 \mathrm{E}-5$ & -0.003016028 & $1.6028 \mathrm{E}-5$ \\
\hline & 0 & 0 & 0.0000000570 & $5.7023 \mathrm{E}-8$ & -0.000014042 & $1.4042 \mathrm{E}-5$ \\
\hline & 0.2 & 0.0030000000 & 0.0029857000 & $1.4299 \mathrm{E}-5$ & 0.002988355 & $1.1644 \mathrm{E}-5$ \\
\hline & 0.4 & 0.0060000000 & 0.0059800185 & $1.9981 \mathrm{E}-5$ & 0.0059910700 & $8.9299 \mathrm{E}-6$ \\
\hline & 0.6 & 0.0090000000 & 0.0089828075 & $1.7192 \mathrm{E}-5$ & 0.008994024 & $5.9752 \mathrm{E}-6$ \\
\hline & 0.8 & 0.0120000000 & 0.0119918316 & $8.1683 \mathrm{E}-6$ & 0.0119971724 & $2.8275 \mathrm{E}-6$ \\
\hline & 1.0 & 0.0150000000 & 0.0150009457 & $9.457 \mathrm{E}-8$ & 0.015001040 & $1.0403 \mathrm{E}-6$ \\
\hline \multirow{11}{*}{0.7} & -1.0 & -0.350000000 & -0.36583575 & $1.5835 \mathrm{E}-2$ & -0.350210888 & $2.1082 \mathrm{E}-4$ \\
\hline & -0.8 & -0.280000000 & -0.27822360 & $1.7763 \mathrm{E}-3$ & -0.280286378 & $2.8637 \mathrm{E}-4$ \\
\hline & -0.6 & -0.210000000 & -0.20836433 & $1.6356 \mathrm{E}-3$ & -0.210322188 & $3.2218 \mathrm{E}-4$ \\
\hline & -0.4 & -0.140000000 & -0.13889262 & $1.1073 \mathrm{E}-3$ & -0.14033638 & $3.3638 \mathrm{E}-4$ \\
\hline & -0.2 & -0.700000000 & -0.06948053 & $5.1946 \mathrm{E}-4$ & -0.070329283 & $3.2928 \mathrm{E}-4$ \\
\hline & 0 & 0 & 0.000027245 & $2.7245 \mathrm{E}-5$ & -0.000301555 & $3.0155 \mathrm{E}-4$ \\
\hline & 0.2 & 0.7000000000 & 0.697120697 & $2.8793 \mathrm{E}-4$ & 0.6974498644 & $2.5501 \mathrm{E}-4$ \\
\hline & 0.4 & 0.1400000000 & 0.139607442 & $3.9255 \mathrm{E}-4$ & 0.139806915 & $1.9308 \mathrm{E}-4$ \\
\hline & 0.6 & 0.2100000000 & 0.209704661 & $2.9533 \mathrm{E}-4$ & 0.209878520 & $1.2147 \mathrm{E}-4$ \\
\hline & 0.8 & 0.2800000000 & 0.279942888 & $5.7111 \mathrm{E}-4$ & 0.279950061 & $4.9938 \mathrm{E}-5$ \\
\hline & 1.0 & 0.3500000000 & 0.350129197 & $1.2919 \mathrm{E}-4$ & 0.3500042976 & $4.2976 \mathrm{E}-6$ \\
\hline
\end{tabular}


Exact solution: $\phi(x, t)=x t$

Example 2

Consider:

$$
\phi(x, t)=f(x, t)+\lambda \int_{-1}^{1} \ln |x-y|\left(\frac{y t}{2}\right)^{2} \mathrm{~d} y+\lambda \int_{0}^{t} t \tau \phi(x, \tau) \mathrm{d} \tau
$$

Exact solution: $\phi(x, t)=\frac{x t}{2}$

\section{Conclusion}

The goal of this work is to study the H-VIE with singular kernel of the second kind. TMM and PNM are successive to solve this equation numerically. As $N$ is increasing, the errors are decreasing. As $t$ is increasing, the errors are increasing.

\section{Conflicts of Interest}

The author declares no conflicts of interest regarding the publication of this paper.

\section{References}

[1] Abdou, M.A. (2003) Fredholm-Volterra Integral Equation with Singular Kernel. Applied Mathematics and Computation, 137, 231-243. https://doi.org/10.1016/S0096-3003(02)00046-2

[2] Al-Bugami, A.A. (2008) Some Numerical Method for Solving Singular and Nonlinear Integral Equation. Umm Al-Qura University, Makkah.

[3] Abdou, M.A., Elsayed, W.G. and Deebs, E.I. (2005) A Solution of a Nonlinear Integral Equation. Applied Mathematics and Computation, 160, 1-14. https://doi.org/10.1016/S0096-3003(03)00613-1

[4] Abdou, M.A. and Hendi, F.A. (2005) Numerical Solution for Fredholm Integral Equation with Hilbert Kernel. The Journal of the Korean Society for Industrial and Applied Mathematics, 9, 111-123.

[5] Al-Bugami, A.A. (2013) Toeplitz Matrix Method and Volterra-Hammerstein Integral Equation with a Generalized Singular Kernel. Progress in Applied Mathematics, 6, 16-42. https://doi.org/10.14419/ijbas.v2i1.601

[6] Abdou, M.A., El-Boria, M.M. and El-Kojok, M.M. (2009) Toeplitz Matriex Method and Nonlinear Integral Equation of Hammerstein Type. Journal of Computational and Applied Mathematics, 223, 765-776. https://doi.org/10.1016/j.cam.2008.02.012

[7] Al-Bugami, A.M. (2013) Error Analysis for Numerical Solution of Hammerstein Integral Equation with a Generalized Singular Kernel. Progress in Applied Mathematics, 6, 1-15. https://doi.org/10.14419/ijbas.v2i1.601

[8] Shahsavaran, A. (2011) Lagrange Functions Method for Solving Nonlinear Fredholm-Volterra Integral Equation. Applied Mathematical Sciences, 5, 2443-2450.

[9] Darwish, M.A. (2004) On Nonlinear Fredholm-Volterra Integral Equations with Hysteresis. Journal Applied Mathematics and Computation, 156, 479-484. https://doi.org/10.1016/j.amc.2003.08.006 
[10] Mirzaee, F. (2017) Numerical Solution of Nonlinear Fredholm-Volterra Integral Equations via Bell Polynomials. Computational Methods for Differential Equations, 5, 88-102.

[11] Raad, S.A. (2005) Some Numerical Methods for Solving Singular Integral Equation. Umm Al-Qura University, Makkah. 